\title{
Soy milk decreased chronic constipation in young children
}

\author{
Iacono G, Cavataio F, Montalto G, et al. Intolerance of cow's milk and chronic constipation in children. N Engl J Med 1998 Oct \\ 15;339:1100-4.
}

\section{Question}

Does soy milk increase stool frequency in young children who have chronic constipation and are being given cows' milk?

\section{Design}

Randomised, controlled, double blind, crossover trial.

\section{Setting}

A paediatric gastroenterology clinic at a university hospital in Italy.

\section{Patients}

65 children $<6$ years of age (mean age 34.6 mo, $55 \%$ girls) who were referred for chronic constipation (chronic fecal retention of 1 bowel movement every 3-15 d often associated with abdominal symptoms). Exclusion criteria were anatomical causes of constipation, constipation caused by another disorder, previous anal surgery, and use of medications associated with constipation. All children were being fed cows' milk, dairy products, or commercial formulas derived from cows' milk. All had unsuccessful treatment with laxatives. Follow up was complete.

\section{Intervention}

Children who were $<15$ months old were given formula (based on cows' milk or soy milk) and older children were given whole cows' milk or soy milk. In the first stage, 33 children were allocated to cows' milk and 32 children were allocated to soy milk for 2 weeks. After a 1 week wash out period, during which use of soy or cows' milk derivatives was unrestricted, children were given the alternate milk for another 2 weeks. Parents were asked to withhold foods that contained milk during the study.
Children with a positive response to soy milk took it for an additional month and then were re-randomised to cows' milk or soy milk for a 2 week period (challenge test).

\section{Main outcome measures}

A positive response defined as $\geqslant 8$ bowel movements during the 2 week study period. Fecal score was also calculated ( 1 for mushy or liquid stools, 2 for soft feces, and 3 for hard stools and difficulty and pain on passing stools).

\section{Main results}

44 of the 65 children $(68 \%)$ had a response while receiving soy milk and none of the children had a response while receiving cows' milk. Children who received cows' milk had a mean of 4 bowel movements during the 2 study weeks and children who received soy milk had a mean of 10 bowel movements $(\mathrm{p}<0.001)$. Children who received soy milk had better fecal scores $(1$ or $2 v 3)(\mathrm{p}<0.001)$ than children who received cows' milk. Of the 44 children in the challenge test, all children who received cows' milk had constipation compared with none of the children who received soy milk. At baseline, children who responded to soy milk had more anal fissures with erythema or edema $(91 \% v 43 \%, \mathrm{p}<0.001)$, symptoms of intolerance to cows' milk $(25 \% v 5 \%, \mathrm{p}=0.05)$, and positive results for immunological tests $(70 \% v 19 \%, \mathrm{p}<0.001)$.

\section{Conclusion}

In young children, constipation was associated with cows' milk; soy milk reduced the constipation.

Source of funding: none stated.

For correspondence: Dr A Carroccio, University of Palermo, via Coffaro 25, I-90124 Palermo, Italy. Fax $+39(0) 916552936$.

\section{Commentary}

Evidence to suggest that childhood constipation may be caused by milk intolerance has appeared in the medical literature from as far back as $1954 .{ }^{1}$ Several studies have suggested that causes of chronic constipation are either psychological or caused by disturbances in intestinal motility. In 1995, however, Iacono et al carried out a study that linked constipation to milk intolerance. ${ }^{2}$ This follow up research from the same authors provides further evidence that constipation is indeed caused by intolerance to the protein found in cows' milk. Immunological tests showed hypersensitivity in nearly three quarters of children with constipation, increasing the possibility that the constipation was related to food intolerance. Mucosal inflammation was more frequent in children with an immune response.
This is an extremely well designed, thorough study. A strength of the study is that the 44 children who had a positive response to soy milk were studied again after a further month on the soy diet. The findings of the study support the authors' theory and are indeed dramatic. It is seldom we find such clear cut results in healthcare research, and we must therefore exercise a certain degree of caution. These children cannot be looked upon as being representative of the general population. They were chosen from a highly specialised centre dealing with the treatment of food allergies, and could be regarded as being preselected by the referring paediatrician.

Notwithstanding that further research needs to be done in other centres to confirm the findings of this study, it would seem that substituting cows' milk with soy milk for a trial period should perhaps be recommended as the first line of treatment before administering large doses of laxatives. The results of this research should be of interest to nurses who deal with children suffering from chronic constipation.

Lesley Ramsay, RGN, RSCN Senior Staff Nurse

Children's Ward, North Tyneside Hospital Tyne and Wear, UK

1 Clein NW. Cows' milk allergy in infants. Pediatr Clin North Am 1954;4:949-62.

2 Iacono G, Carroccio A, Cavataio F, et al. Chronic constipation as a symptom of cow milk allergy.J Pediatr 1995;1 26:34-9. 